\title{
A single distance sample of molecular outflows from high-mass young stellar objects
}

\author{
N. A. Ridge ${ }^{1,2}$ and T. J. T. Moore ${ }^{1}$ \\ 1 Astrophysics Research Institute, Liverpool John Moores University, Twelve Quays House, Egerton Wharf, \\ Birkenhead CH41 1LD, UK \\ 2 Current Address: FCRAO, 619 Lederle Graduate Research Center, University of Massachusetts, \\ Amherst, MA 01003, USA
}

Received 11 June 2001 / Accepted 22 August 2001

\begin{abstract}
We have made ${ }^{12} \mathrm{CO} 2-1$ and 1-0 maps of eleven molecular outflows associated with intermediate to high-mass young stellar objects (YSOs) in order to establish whether the correlations between outflow parameters and source bolometric luminosity hold in the high-mass regime. It is important to consider the effects of Malmquisttype biases when looking at high-mass YSOs, as they are generally much more distant than their low mass counterparts. We therefore chose only objects located at $\sim 2 \mathrm{kpc}$. We find that the relations show much more scatter than is seen in similar studies of low-mass YSOs. We also find that the mass-spectrum is significantly steeper in high-mass outflows, indicating a larger mass-fraction at lower velocities, a low collimation factor $(\sim 1-2)$ and no Hubble-like relationship.
\end{abstract}

Key words. stars: formation - stars: winds, outflows - ISM: jets and outflows - ISM: molecules

\section{Introduction}

It has been well established that outflows represent an important evolutionary stage in the formation of low-mass stars (Bachiller 1996), but systematic studies to identify bipolar outflows from massive YSOs have only begun much more recently. There is now growing evidence that bipolar molecular outflows are ubiquitous with YSOs of all masses. Shepherd \& Churchwell (1996a) searched for high velocity (HV) CO wings toward $\sim 120$ high-mass star forming regions, and found that HV gas was present in $\sim 90 \%$ of sources, indicating that molecular outflows are also a common property of high-mass YSOs. Most luminous YSOs seem to have outflows with much wider opening angles than their low-mass counterparts (Richer et al. 2000), but this may be due to the poor resolution that the known flows have been observed with, and/or the poor statistics available due to the small number of flows which have been studied. It is difficult to make predictions about the general properties of high-mass flows due to the selection effects inherent in the small existing samples.

Shepherd \& Churchwell (1996b) determined the energetics of 5 massive YSO outflows and showed that they fit well onto the correlation between outflow force $\left(F_{\mathrm{CO}}\right.$, sometimes referred to as momentum flux) and

Send offprint requests to: N. A. Ridge,

e-mail: naomi@fcrao.umass.edu bolometric luminosity presented by e.g. Cabrit \& Bertout (1992; hereafter CB). Henning et al. (2000) studied three more massive outflows with similar results. However, we have shown (Moore \& Ridge, in preparation) that these correlations, which use heterogeneous, unconstrained samples, may be contaminated by biases due to source distance. We have therefore undertaken a program of observations to study a larger sample of high-mass outflows at a single distance of $2 \mathrm{kpc}$, with consistent sensitivity, spatial resolution and analysis. The sample is discussed in detail in Sect. 2, then the observations and results are presented in Sects. 3 and 4. Sections 5 and 6 contain the discussion and conclusions respectively.

\section{The sample}

We selected a base sample of 25 objects from the compilation of $\mathrm{Wu}$ et al. (1996), with the selection criteria $L_{\text {bol }} \geq 10^{2} L_{\odot}$, and distance $2.0 \pm 0.3 \mathrm{kpc}$. This full sample was reduced to a final list of 11 targets by rejecting objects known to have very weak and diffuse flows (e.g. AFGL 2591), complex and possibly multiple flows (e.g. DR 21), and flows with little obvious bipolarity (i.e. flows which may be pole-on, for which it is difficult to define the flow extent). Table 1 lists the selected outflows. All of these flows have been previously observed with low resolution and with different sensitivities, but few have 
Table 1. Single distance sample of outflow sources. Columns 2 and 3 give the positions of the sources. All offsets quoted throughout this paper are given relative to these positions. $V_{\mathrm{LSR}}$ is the velocity of the ambient cloud material relative to the local standard of rest. $L_{\mathrm{bol}}$ is the bolometric luminosity of the source believed to be the driving source for the outflow. Luminosities given by $\mathrm{Wu}$ et al. (1996) were checked by referring to the original paper, and found to be unreliable in several cases.

\begin{tabular}{ccccccc}
\hline Source & $\begin{array}{c}\text { RA (1950) } \\
\text { hh:mm:ss }\end{array}$ & $\begin{array}{c}\text { Dec (1950) } \\
\text { dd:mm:ss }\end{array}$ & $\begin{array}{c}L_{\text {bol }} \\
L_{\odot}\end{array}$ & $\begin{array}{c}D \\
\mathrm{kpc}\end{array}$ & $\begin{array}{c}V_{\mathrm{LSR}} \\
\mathrm{km} \mathrm{s}^{-1}\end{array}$ & $\operatorname{Refs}^{b}$ \\
\hline NGC 6334B & $17: 17: 18$ & $-35: 48: 00$ & $4.00 \times 10^{5}$ & 1.7 & 0 & 1 \\
NGC 6334I & $17: 17: 34$ & $-35: 43: 47$ & $8.00 \times 10^{4}$ & 1.74 & -7.5 & 1 \\
GGD 27 & $18: 16: 13$ & $-20: 48: 43$ & $2.0 \times 10^{4}$ & 1.7 & +12 & 1 \\
S88 B & $19: 44: 40$ & $+25: 05: 30$ & $1.80 \times 10^{5}$ & 2.0 & +21 & 1 \\
IRAS 19550 & $19: 54: 59$ & $+32: 48: 29$ & $1.50 \times 10^{2}$ & 2.0 & +12 & 1 \\
IRAS 20188 & $20: 18: 51$ & $+39: 28: 18$ & $1.26 \times 10^{4}$ & 2.0 & +2 & 1 \\
W75 N & $20: 36: 51$ & $+42: 27: 20$ & $1.40 \times 10^{5}$ & 2.0 & +9 & 1,2 \\
W3 IRS5 & $02: 21: 53$ & $+61: 52: 21$ & $1.10 \times 10^{6}$ & $2.3^{a}$ & -40 & 1 \\
AFGL 437 & $03: 03: 32$ & $+58: 19: 37$ & $2.40 \times 10^{4}$ & 2.0 & -39 & 1,3 \\
AFGL 5142 & $05: 27: 28$ & $+33: 45: 37$ & $3.80 \times 10^{3}$ & 1.8 & -4 & 1,4 \\
AFGL 5157 & $05: 34: 33$ & $+31: 57: 40$ & $5.50 \times 10^{3}$ & 1.8 & -18 & 1 \\
\hline
\end{tabular}

${ }^{a}$ Imai et al. (2000) find a distance to W3 IRS5 of $1.8 \pm 0.14 \mathrm{kpc}$ based on maser observations. ${ }^{b}$ Refs: 1 . Wu et al. (1996), 2. Moore (1989), 3. Weintraub \& Kastner (1996), 4. Hunter et al. (1995).

been previously mapped in any detail. The sources are selected from an existing, heterogeneous catalogue of outflow sources, and therefore may not be truly representative of the population properties. However, by selecting objects with a range of luminosities, all at the same distance, biases should be reduced to minimum. The bolometric luminosities listed in Table 1 are generally based on IRAS fluxes and thought to be reliable.

\section{Observations and data analysis}

Observations were carried out between 1998 August and 1999 June at the $15 \mathrm{~m}$ James Clerk Maxwell Telescope (JCMT) on Mauna Kea, Hawaii and at the NRAO $12 \mathrm{~m}$ Radio Telescope (NRAO) at Kitt Peak, Arizona.

\subsection{JCMT observations}

We obtained ${ }^{12} \mathrm{CO} J=2 \rightarrow 1$ "flexibly-scheduled" observations between 1998 August and 1999 May and 4 scheduled nights of observations in 1999 July. At the observing frequency of $230 \mathrm{GHz}$ the half-power beam width of the telescope is $21^{\prime \prime}$. We used the DAS spectrometer with 1657 channels, providing an effective velocity resolution of $0.4 \mathrm{~km} \mathrm{~s}^{-1}$. The system temperatures during the observations varied between $300 \mathrm{~K}$ and $500 \mathrm{~K}$ depending on the weather. Pointing and focus checks were carried out regularly on planets or bright galactic sources. Pointing was found to vary by less than $5^{\prime \prime}$ during the runs.

The observations were carried out in raster-mapping mode with a grid spacing of $10^{\prime \prime}$ producing fully sampled maps. In this mode, the telescope observes one "off" position per row of on-source observations. Maps were repeated 4 times to obtain the required rms sensitivity of $0.2 \mathrm{~K}$ per $1 \mathrm{~km} \mathrm{~s}^{-1}$ resolution element. The reference positions were checked for the presence of $\mathrm{CO}$ emission by single position-switched observations.

The data were calibrated using the standard chopperwheel technique, so the intensity scale for all JCMT spectral data presented here is expressed in units of $T_{\mathrm{A}}^{*}$ (Kutner \& Ulich 1981) which is the source antenna temperature corrected for atmospheric and rearward spillover losses. This is converted to radiation temperature, by correcting for the forward spillover efficiency $\eta_{\mathrm{fss}}$. Single positionswitched observations of a standard source (W3) were also obtained so that the data could be compared with the NRAO observations.

\subsection{NRAO Observations}

We obtained ${ }^{13} \mathrm{CO} J=2-1$ and ${ }^{12} \mathrm{CO} J=1-0$ observations at the NRAO $12 \mathrm{~m}$ telescope during scheduled runs in 1998 November, 1999 March and 1999 April. These data were necessary in order to investigate variations in optical depth and excitation which can significantly affect the masses derived via the LTE approximation.

The telescope has a half-power beam width of $27^{\prime \prime}$ at $230 \mathrm{GHz}$ and $55^{\prime \prime}$ at $115 \mathrm{GHz}$. At $230 \mathrm{GHz}$ the dual-channel single-beam SIS receiver was used with two filter bank spectrometers in parallel, each with 128 channels. The filter banks were used at $500 \mathrm{kHz}$ and $1 \mathrm{MHz}$ bandwidths providing a spectral resolution of $0.7 \mathrm{~km} \mathrm{~s}^{-1}$ and $1.3 \mathrm{~km} \mathrm{~s}^{-1}$ respectively at $230 \mathrm{GHz}$. System temperatures varied during the runs, and channel 2 of the receiver achieved consistently worse performance during the observations.

The observations were made in On-the-Fly mapping mode. As at the JCMT, the chopper wheel calibration method was used, yielding intensities on the $T_{\mathrm{A}}^{*}$ scale.

The mapped fields were the same size as the JCMT fields, plus a 30" "ramp-up" distance added to the end 
Table 2. ${ }^{12} \mathrm{CO}$ flux at the positions of maximum integrated intensity of the red- and blue-shifted emission.

\begin{tabular}{ccc}
\hline Source & $\begin{array}{c}F_{\text {peak }}(\text { red }) \\
\mathrm{K} \mathrm{km} \mathrm{s}^{-1}\end{array}$ & $\begin{array}{c}F_{\text {peak }}(\text { blue }) \\
\mathrm{K} \mathrm{km} \mathrm{s}^{-1}\end{array}$ \\
\hline NGC 6334I & 290 & 640 \\
NGC 6334B & 214 & 252 \\
S88 B & 130 & 240 \\
AFGL 437 & 130 & 100 \\
AFGL 5142 & 195 & 163 \\
AFGL 5157 & 168 & 81 \\
W3 IRS5 & 680 & 656 \\
GGD 27 & 100 & 190 \\
IRAS 19550 & 40 & 36 \\
IRAS 20188 & 222 & 245 \\
W75 N & 183 & 60 \\
\hline
\end{tabular}

of each row. A row spacing of $8^{\prime \prime}$ and row scanning rate $44^{\prime \prime} \mathrm{s}^{-1}$ were used, resulting in fully-sampled maps. Maps were repeated, scanning alternately in the RA and Dec directions to remove artifacts in the scanning direction, and later combined and gridded together to improve signal-tonoise in the final map.

\subsection{Analysis}

A local thermal equilibrium (LTE) approximation was used to convert the ${ }^{12} \mathrm{CO}$ intensities into column densities, using the ${ }^{13} \mathrm{CO} 2-1$ data to correct for optical depth variations with velocity and position, assuming a solarsystem isotopic abundance ratio of 89 . Smaller values of this ratio have been observed by e.g. Langer \& Penzias (1990) but this will affect the derived masses by less than a factor of two (Ridge 2000). ${ }^{12} \mathrm{CO} 1-0$ data was additionally used to calculate excitation temperatures to improve the analysis. ${ }^{12} \mathrm{CO}$ column densities were then converted to masses using a standard $\left[\frac{X_{\mathrm{CO}}}{X_{\mathrm{H}_{2}}}\right]$ Galactic abundance ratio.

Flow dynamics were derived following the method of Cabrit \& Bertout (1992). A full description of the data reduction and analysis procedure is given in Ridge (2000).

\section{Observational results}

\subsection{Morphology}

Maps of the ${ }^{12} \mathrm{CO} J=2-1$ integrated intensity from JCMT are shown in Figs. 1 and 2. Blue-shifted emission is shown as dashed lines, and red-shifted emission is shown as solid lines. Contour levels are as given in the captions, with the peak-flux (integrated ${ }^{12} \mathrm{CO}$ flux at the position of maximum integrated intensity) summarised in Table 2. The integration limits for the red and blue shifted gas are summarised in Table 3. In general the flows are not wellcollimated, but bipolar structure is evident in most cases. We discuss each object individually below.
Table 3. The ranges of red and blue shifted emission over which the integration was carried out. Velocities are LSR.

\begin{tabular}{ccc}
\hline Source & $\begin{array}{c}\Delta V_{\text {red }} \\
\mathrm{km} \mathrm{s}^{-1}\end{array}$ & $\begin{array}{c}\Delta V_{\text {blue }} \\
\mathrm{km} \mathrm{s}^{-1}\end{array}$ \\
\hline NGC 6334I & $2.5 \rightarrow 40.0$ & $-40.0 \rightarrow-10.0$ \\
NGC 6334B & $3.0 \rightarrow 6.0$ & $-18.0 \rightarrow-6.0$ \\
S88 B & $24.5 \rightarrow 31.0$ & $7.5 \rightarrow 20.0$ \\
AFGL 437 & $-37.0 \rightarrow-25.0$ & $-48.0 \rightarrow-43.0$ \\
AFGL 5142 & $-3.0 \rightarrow 6.0$ & $-17 \rightarrow-5$ \\
AFGL 5157 & $-16.0 \rightarrow-3.0$ & $-30.0 \rightarrow-21.5$ \\
W3 IRS5 & $-39.0 \rightarrow-14.0$ & $-61.0 \rightarrow-43.0$ \\
GGD 27 & $14.5 \rightarrow 20.0$ & $1.5 \rightarrow 10.5$ \\
IRAS 19550 & $14.0 \rightarrow 21.0$ & $5.5 \rightarrow 11.5$ \\
IRAS 20188 & $4.0 \rightarrow 16.0$ & $-20.0 \rightarrow-2.0$ \\
W75 N & $15.0 \rightarrow 32.5$ & $-30.0 \rightarrow-18.0$ \\
\hline
\end{tabular}

\subsubsection{NGC 6334I}

NGC 6334 is a large complex of HII regions and an associated molecular cloud in the southern Galactic plane, containing numerous sites of recent or ongoing star formation. NGC 6334I is the brightest far-infrared source in the northern part of NGC 6334 (Loughran et al. 1986) and is the dominant source at millimetre and sub-millimetre wavelengths (Sandell 2000). The high-velocity outflow was first detected by Bachiller \& Cernicharo (1990). NGC 6334I is also a rich source of molecules (Bachiller \& Cernicharo 1990) and has water, methanol and $\mathrm{OH}$ masers associated with it. Three ammonia masers have also been detected near the ends of the outflows (Kraemer \& Jackson 1995).

The integrated contour map of the NGC 6334I outflow is shown in Fig. 1. There are two distinct flows, indicated by two clearly separated lobes present in the red-shifted emission (solid contours). It is less clear in the blue-shifted emission, but there seems to be an extension to the northwest which could be a second flow lobe. The second, lessdominant flow seems to be centred to the west of the stronger flow, and probably originates from a second object. This is confirmed by McCutcheon et al. (2000) who detect this feature in both ${ }^{12} \mathrm{CO} 3-2$ and in CS, and assign it the name $I(\mathrm{NW}) . I(\mathrm{~N})$ is a third object in this region, located $\sim 100^{\prime \prime}$ north of NGC 6334I. The bottom edge of the outflow from this source can be seen on the northern edge of the frame. Outflow dynamics were calculated from the dominant outflow, closest to the IRAS source. Extremely high velocity emission is seen in the spectra of this source and there is a sharp absorption feature at $V_{\mathrm{LSR}}+6.5 \mathrm{~km} \mathrm{~s}^{-1}$. This is a real line in the same sideband, not due to emission in the off position and is also seen in $J=3-2$ emission by McCutcheon et al. (2000) widespread over the northern part of the NGC 6334 complex, suggesting that a foreground cloud at a lower temperature may be obscuring the radiation from NGC 6334I. 

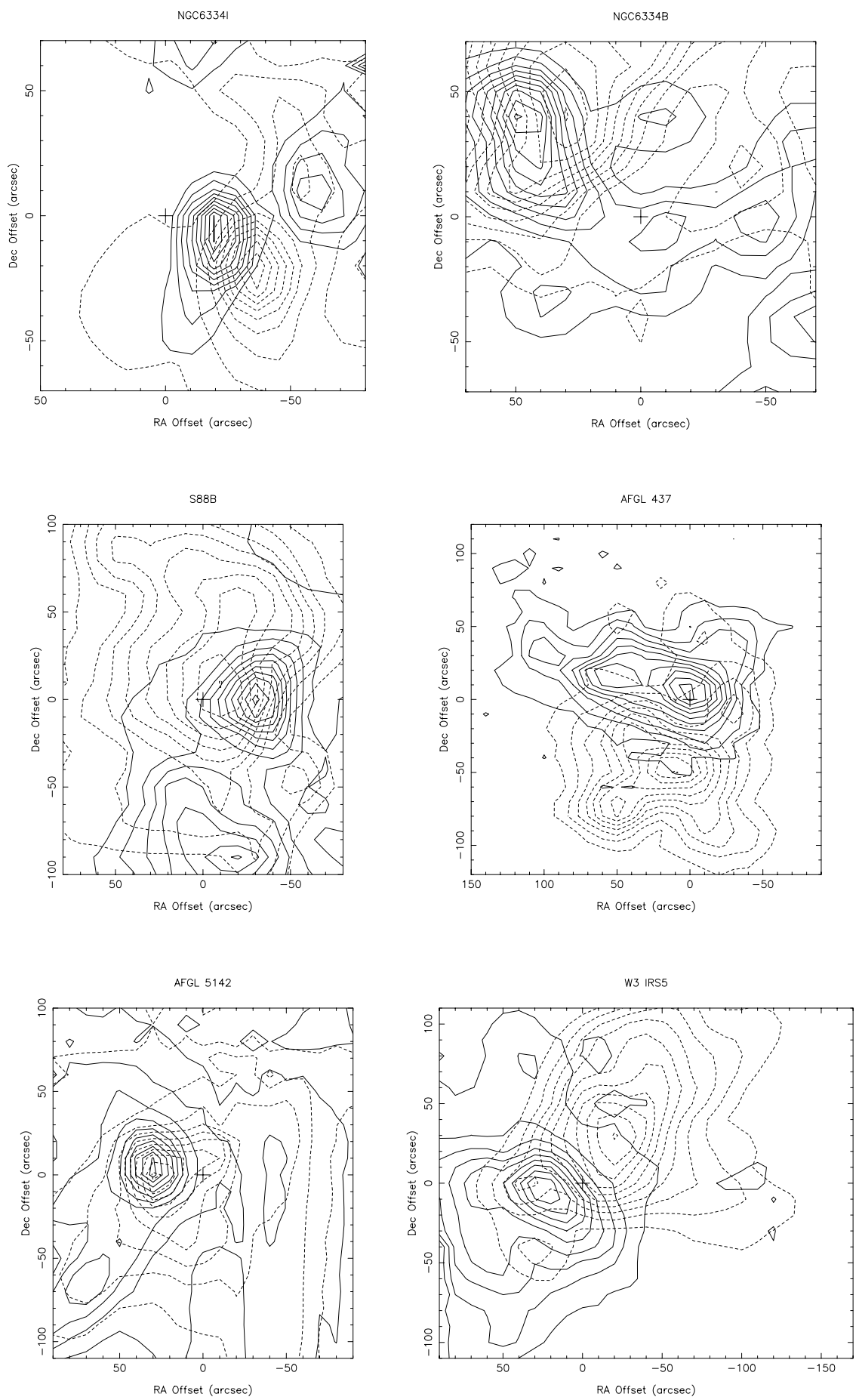

Fig. 1. Maps of the integrated ${ }^{12} \mathrm{CO} J=2-1$ emission from 6 sources made at the JCMT with $21^{\prime \prime}$ resolution. Contours are at $10 \%$ intervals in all cases. Blue-shifted material is indicated by dashed contours and red-shifted material by solid contours. Crosses indicate the position of the infrared source associated with each of the flows. Velocity integration ranges are given in Table 3.

\subsubsection{NGC 6334B}

The second source in the NGC 6334 complex, NGC 6334B was observed by Phillips \& Mampaso (1991). Their observations centred on the $\mathrm{CO}$ peak position of Dickel et al. (1977), and they did not find a bipolar structure in the high-velocity gas. However, there is an extremely luminous FIR source $\left(L \sim 4 \times 10^{5} L_{\odot}\right.$; Loughran et al. 1986) and H II region $40^{\prime \prime}$ to the north of this position, and our maps were therefore offset north $40^{\prime \prime}$ from the $\mathrm{CO}$ peak position.

The integrated contour map of the NGC 6334B outflow is shown in Fig. 1. This flow shows two lobes well offset to the north-east from the centre of the map. The peak 
emission of the two lobes is separated by one beam width. The blue lobe may stretch beyond the map boundary to the north. The flow lobes are not well collimated. Both the ${ }^{12} \mathrm{CO}$ and ${ }^{13} \mathrm{CO}$ spectra show peaks at 0 and $-5 \mathrm{~km} \mathrm{~s}^{-1}$, indicating two separate cloud components, and the spectrum from the red peak position shows a possible third peak at $3 \mathrm{~km} \mathrm{~s}^{-1}$. This is clearly a complex region.

\subsubsection{S88 B}

S88 B is a compact H II region located within the S88 cloud. Single-dish molecular line observations show that the S88 B complex is embedded within a molecular cloud core of relatively low mean density, $n\left(\mathrm{H}_{2}\right) \sim 6 \times 10^{3} \mathrm{~cm}^{-3}$, out of which the exciting stars of the $\mathrm{H}$ II regions presumably formed (e.g. Phillips \& Mampaso 1991 and Refs. therein). The outflow was mapped by Phillips \& Mampaso (1991) with $30^{\prime \prime}$ resolution.

The integrated contour map of the outflow from S88 B is shown in Fig. 1. Two lobes are clearly visible, with the red-shifted lobe (solid contours) appearing more collimated than the blue-shifted lobe (dashed contours). Two distinct peaks are present in the red-shifted emission, but the blue-shifted emission gives no indication of a second bipolar outflow to the south.

\subsubsection{AFGL 437}

AFGL 437 is a compact cluster (Kleinmann et al. 1977) containing at least 3 highly polarised YSOs (Dyck \& Lonsdale 1979). Asymmetric profiles were detected near this source by Schneps et al. (1978), but were not initially interpreted as a bipolar outflow. Arquilla \& Goldsmith (1984) made observations at 50" resolution and a higher sensitivity and interpreted the profiles as produced by a large-scale bipolar outflow, with the red and blue lobes located at the north and south of the central cluster, and their maxima separated by $\sim 1.2^{\prime}$. Gómez et al. (1992) mapped the region at higher resolution and found a compact outflow with a very low degree of collimation.

The integrated contour map of AFGL 437 is shown in Fig. 1. This is a poorly collimated compact flow. However bipolar structure is still evident, with the blue lobe stretching south and the red lobe extending to the north. The north lobe particularly appears elongated perpendicular to the outflow axis. The AFGL 437 infra-red cluster, indicated in the diagram by the cross is not at the centre of the outflow. We suggest that the contour pattern here is consistent with the base of a very wide parabolic outflow in a clumpy medium. Weintraub \& Kastner (1996) use infrared polarisation data to explain the unusual morphology of this CO outflow by the presence of an obstruction to the north of AFGL 437 which will defocus and deflect the flow. It may also be due to the position of the cluster near to the edge of the molecular cloud (Gómez et al. 1992).

\subsubsection{AFGL 5142}

The star-forming region AFGL 5142 contains an IRAS source with a bolometric luminosity $3.8 \times 10^{3} L_{\odot}$ (Carpenter et al. 1990). The CO outflow was found by Snell et al. (1988) with moderate resolution observations. Its radio continuum flux density is consistent with a B2ZAMS star, assuming optically thin free-free emission (Torrelles et al. 1992a).

There is a cluster of infrared sources in the centre of the region with a radius of $0.3 \mathrm{pc}\left(30^{\prime \prime}\right.$ at $2 \mathrm{kpc}$; Hunter et al. 1995), many of the sources showing a strong infrared excess. Two cluster members, IRS 1 which has the strongest IR excess and IRS 2, the brightest member at $K$ band (2.7 magnitudes brighter than IRS 1 ) have received the most attention in previous studies. IRS 2 is at the centre of the large scale outflow (Hunter et al. 1995) and also coincides with the IRAS point source position (marked with a cross in the figure). Hunter et al. suggest that IRS 2 is in an advanced stage of protostellar evolution, in a region where the molecular cloud has been dispersed. IRS 1 is thought to be in a much earlier evolutionary phase, coinciding with an unresolved thermal continuum source, a cluster of $\mathrm{H}_{2} \mathrm{O}$ masers and a small dense molecular cloud core.

The integrated contour map of AFGL 5142 is shown in Fig. 1. This is another relatively compact flow, with the blue and red peaks superimposed suggesting a flow at a small angle to the line of sight. This flow is poorly collimated, and Hunter et al. (1995) suggest it may be multi-polar, with two flows orientated almost perpendicular to one another. Our observations do not support this suggestion.

\subsubsection{W3 IRS5}

The source IRS 5 in the W3 giant molecular cloud was discovered by Wynn-Williams et al. (1972). It has a very high luminosity $\left(\sim 10^{6} L_{\odot}\right)$, extremely steep spectrum and a very deep silicate absorption feature at $9.8 \mu \mathrm{m}$ (Willner 1977). The outflow was first identified by Bally \& Lada (1983).

The integrated contour map of W3 IRS5 is shown in Fig. 1. Although relatively poorly collimated, bipolar structure is clearly evident in this outflow. Emission is present out to $\gtrsim 20 \mathrm{~km} \mathrm{~s}^{-1}$ in this flow. This source shows strong self-absorption, especially noticeable in the spectrum from the blue lobe, where the line centre, indicated by the peak of the ${ }^{13} \mathrm{CO}$ spectrum, is almost totally selfabsorbed.

\subsubsection{AFGL 5157}

AFGL 5157, situated in the Perseus arm (Roberts 1972), is an extensively studied star-forming region. Two optical reflection nebulae, NGC 1985 and GM 39, have been observed in AFGL 5157 (Higgs 1971; Torrelles et al. 1992a). Near-infrared observations by Torrelles et al. (1992b) 

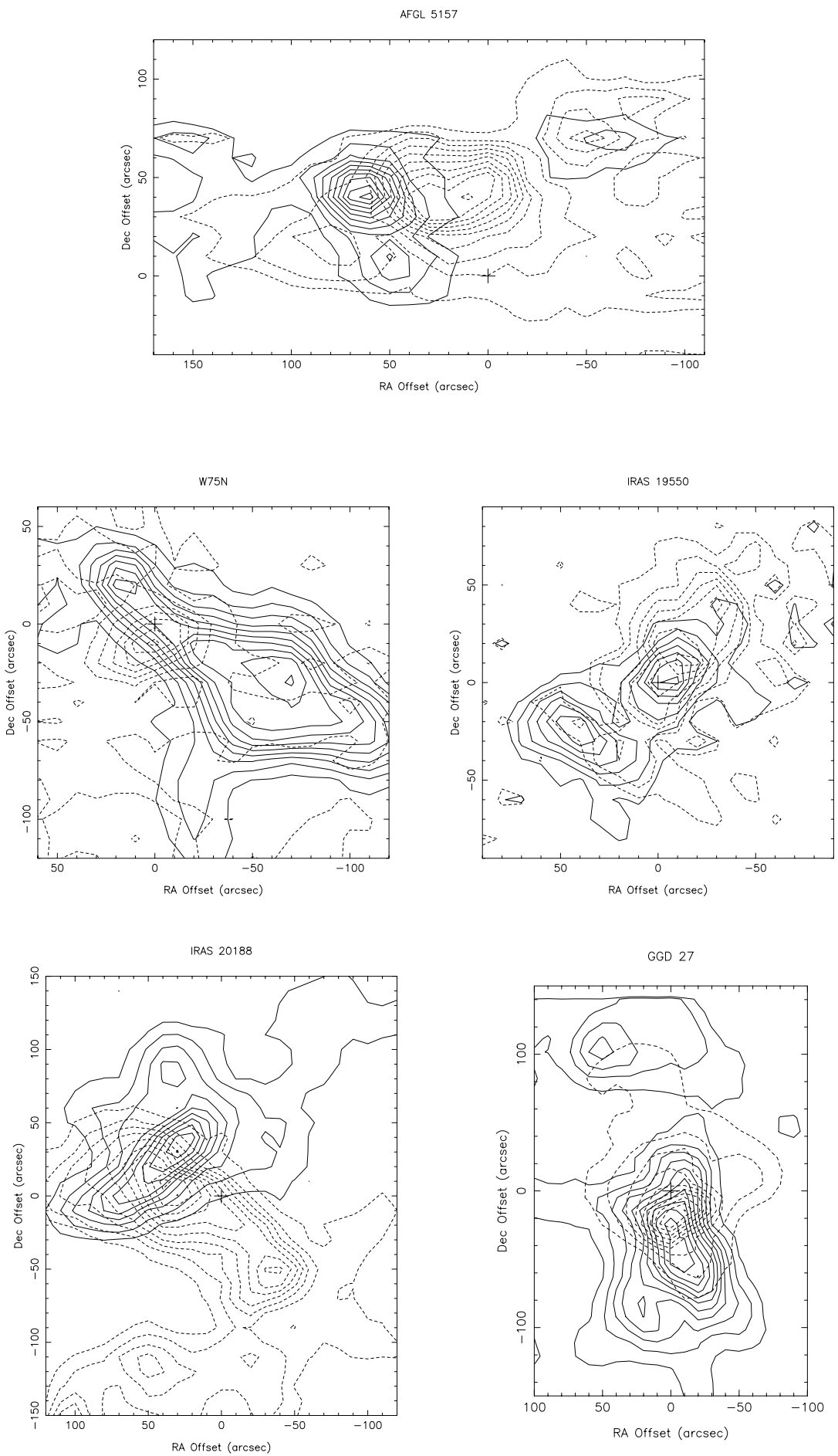

Fig. 2. Maps of the integrated ${ }^{12} \mathrm{CO} J=2-1$ emission from 5 sources made at the JCMT with $21^{\prime \prime}$ resolution. Contours are at $10 \%$ intervals in all cases. Blue-shifted material is indicated by dashed contours and red-shifted material by solid contours. Crosses indicate the position of the infrared source associated with each of the flows. Integration limits for the red and blue wings are given in Table 3.

presented two infrared sources, IRS 1 and 2. IRS 1 appears to be associated with the infrared source IRAS $05345+3157$, which has a far-infrared luminosity of $5.5 \times 10^{3} L_{\odot}($ Snell et al. 1988). IRS 2 is located close to the reflection nebula GM 39. In the $K^{\prime}$ imaging survey by Hodapp (1994), a cluster of stars can be seen in the IRS 1 position associated with dispersed nebulosity.
The IRAS source $05345+3157$ is not thought to be the powering source of the outflow in AFGL 5157, as it is located $\sim 1^{\prime}$ southwest of the outflow and molecular core centre (Verdes-Montenegro et al. 1989). Torrelles et al. (1992b) suggest that the outflow is in fact powered by a deeply embedded source at the centre of the ammonia core and associated with the strong $\mathrm{H}_{2} \mathrm{O}$ maser. 
The integrated contour map of AFGL 5157 is shown in Fig. 2. This flow shows a high degree of collimation and bipolarity with weak extensions stretching for an arcminute to the east and west. The majority of the emission is however concentrated close to central source. There may be a weak second flow located to the north-west.

\subsubsection{W75 N}

$\mathrm{W} 75 \mathrm{~N}$ is a well studied active star-forming region containing compact $\mathrm{H}$ II regions, $\mathrm{OH}$ and water masers, $2 \mu \mathrm{m}$ emission and about 40 embedded sources, as well as the well-known CO outflow (Moore et al. 1991a; Moore et al. 1991b). It is thought to be forming several B-type stars (Hunter et al. 1994). The source associated with IRS 1 and $\mathrm{H}$ II region "B", with a luminosity of $\sim 1.4 \times 10^{5} L_{\odot}$ (Moore et al. 1991a) is thought to be driving the outflow (Moore et al. 1991b). HiI(B) has since been resolved into three subcomponents by Hunter et al. (1994).

The integrated contour map of $\mathrm{W} 75 \mathrm{~N}$ is shown in Fig. 2. There is a well collimated red-shifted lobe which stretches south west from the source. It appears to start very narrow, then open up as it gets further from the source.

It is more difficult to determine the morphology of the blue-shifted lobe, as the spectra are contaminated by emission from the nearby cloud containing the object DR 21 which has an LSR velocity of $-3 \mathrm{~km} \mathrm{~s}^{-1}$ and overlaps the W75 cloud over most its extent (Dickel et al. 1978). The ${ }^{13} \mathrm{CO}$ spectra show the two separate objects clearly, but it is impossible to determine how much of the ${ }^{12} \mathrm{CO}$ emission is from the wings of $\mathrm{W} 75 \mathrm{~N}$ and how much from DR 21 . The peak at an offset of $(0,-15)$ arcseconds is strongest at $\sim+3 \mathrm{~km} \mathrm{~s}^{-1}$ and is therefore likely to be due to the outflow. However, this peak is compact, showing virtually no extension along the axis of the red outflow lobe. Davis et al. (1998) suggested that the small extent of the blue lobe is probably due to environmental asymmetry, with little molecular gas to the north-east of the source, and supported by the fact that the asymmetry of the flow is not reflected in $\mathrm{H}_{2}$ observations. Due to the unusual nature of the blue-lobe of this flow, all calculations of flow properties were made by scaling from the red-lobe values.

\subsubsection{IRAS 19550}

IRAS 19550 was identified as a protostellar object from its outflow by Koo et al. (1994). This object appears to be forming in isolation at the tip of a large filamentary cloud. IRAS 19550 shows $K$-band emission extended along the east-west direction, and $I$ and $R$ band images show that the source is made up of two weak peaks shifted symmetrically from the $K$-band peak, probably due to scattered stellar light (Koo et al. 1994).

The integrated contour map of IRAS 19550 is shown in Fig. 2. This flow is the most compact of those we studied. There is also some indication of a second flow located to the south-west of the source and at right angles to the known flow, particularly evident in the red (solid) contours. The source has very weak ${ }^{12} \mathrm{CO}$ emission.

\subsubsection{IRAS 20188}

IRAS 20188, a compact molecular cloud identified in a survey for molecular line emission from IRAS sources by Richards et al. (1987), is located in the Cygnus region and is not obviously associated with any known stellar cluster or H II region. The outflow was first identified by Little et al. (1988).

The integrated contour map of IRAS 20188 is shown in Fig. 2. The morphology of this flow is difficult to decipher. There appear to be two well-collimated lobes at almost 90 degrees to each other, both containing at least two distinct peaks, which may suggest a multiple flow or alternatively may be a result of multiple outflow episodes. One explanation of this flow is that it is the base of a very wide parabolic flow with an asymmetric distribution of material.

\subsubsection{GGD 27}

GGD 27 was originally catalogued as an optical candidate Herbig-Haro object (Gyulbudaghian et al. 1978), but has now been determined to be the centre of a highly active star-forming region. It contains numerous nearinfrared point sources and extensive reflection nebulosity (Yamashita et al. 1987). The deeply embedded object GGD 27-ILL is thought to be the driving source of the outflow (Aspin et al. 1994), and the nearby source IRS 2 is a knot of dust heated by the the hot circumstellar dust around GGD 27-ILL.

The integrated contour map of GGD 27 is shown in Fig. 2. There are two lobes well separated in velocity (blue lobe integrated between $1.5 \mathrm{~km} \mathrm{~s}^{-1}$ and $10.5 \mathrm{~km} \mathrm{~s}^{-1}$, red lobe integrated between $14.5 \mathrm{~km} \mathrm{~s}^{-1}$ and $20.0 \mathrm{~km} \mathrm{~s}^{-1}$ ), although they are superimposed in space, with the blue lobe stretching to the north, while the red lobe stretches southwards. The red lobe (solid contours) appears slightly more collimated than the blue lobe (dashed contours).

\subsection{Outflow properties}

\subsubsection{Flow sizes and dynamical timescales}

The sizes of the flow lobes were determined from the contour maps by measuring the distance in arcsec between the adopted centre of the flow and the $10 \%$ contour in the direction of the flow axis, where the flow axis was defined as the straight line joining the blue and red emission peaks. The values for the red and blue lobes were then averaged and converted to a physical size in parsec using the distances to the sources listed in Table 1 . The dynamical timescale, $t_{\mathrm{dyn}}$ was then calculated by dividing the flow size by the intensity-weighted velocity. It should be noted however that the dynamical timescale may not be a 
Table 4. Flow properties.

\begin{tabular}{ccccccc}
\hline Object & $\begin{array}{c}\text { Flow Mass } \\
M_{\odot}\end{array}$ & $\begin{array}{c}F_{\mathrm{CO}} \\
M_{\odot} \mathrm{km} \mathrm{s}^{-1} \mathrm{yr}^{-1}\end{array}$ & $\begin{array}{c}L_{\mathrm{CO}} \\
L_{\odot}\end{array}$ & $\begin{array}{c}\text { Flow size } \\
\mathrm{pc}\end{array}$ & $\begin{array}{c}\text { Flow age } \\
\times 10^{4} \mathrm{yr}\end{array}$ & $\begin{array}{c}\dot{M}_{\text {flow }} \\
M_{\odot} \mathrm{yr}^{-1}\end{array}$ \\
\hline AFGL 5157 & 672 & 131 & 217 & 0.69 & 2.49 & 0.027 \\
AFGL 5142 & 1082 & 10.9 & 5.47 & 0.80 & 3.28 & 0.033 \\
AFGL 437 & 620 & 235 & 750 & 1.36 & 5.77 & 0.011 \\
S88 B & 867 & 103 & 222 & 1.12 & 4.68 & 0.019 \\
GGD 27 & 407 & 14.7 & 15.8 & 1.44 & 7.63 & 0.0053 \\
W3 IRS5 & 2016 & 756 & 2594 & 1.73 & 3.60 & 0.056 \\
NGC 6334B & 2344 & 14.1 & 6.2 & 0.69 & 2.82 & 0.083 \\
NGC 6334I & 3317 & 76.6 & 71.9 & 0.74 & 0.60 & 0.55 \\
IRAS 20188 & 2166 & 2.66 & 1.10 & 1.67 & 4.54 & 0.048 \\
IRAS 19550 & 5.77 & 0.29 & 0.35 & 0.58 & 3.67 & 0.00016 \\
W75 N & 548 & 35.2 & 46.4 & 1.23 & 1.93 & 0.028 \\
\hline
\end{tabular}
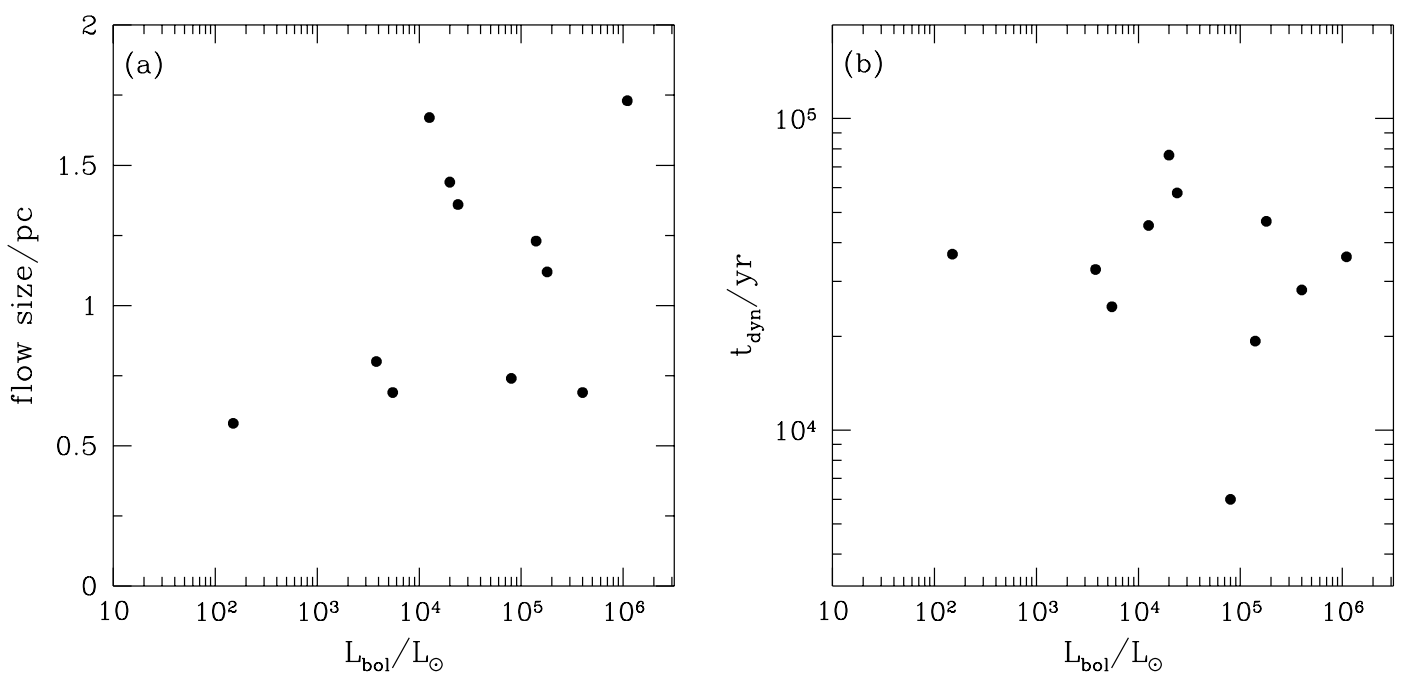

Fig. 3. a) Flow size and b) "Dynamical timescale"versus bolometric luminosity.

true "age" of the flow (see e.g. Padman et al. 1997 for a discussion), particularly for sources like AFGL 437 where there is an obstruction or AFGL 5142 where the lobes are overlapping. The flow sizes and dynamical timescales are listed in Table 4. The flows are all of the order of $1 \mathrm{pc}$ in size. Both $R_{\text {flow }}$ and $t_{\text {dyn }}$ are uncorrelated $(<1 \sigma)$ with bolometric luminosity (Fig. 3).

\subsubsection{Flow mass and energy}

Table 4 lists the dynamical age, mass, CO momentum flux $\left(F_{\mathrm{CO}}\right)$ and power $\left(L_{\mathrm{CO}}\right)$ we have derived for all the objects in the sample, following the method of Cabrit \& Bertout (1992), with corrections applied for variations in optical depth with velocity and position in the flow (see Shepherd \& Churchwell 1996b). A full discussion of the procedure is given in Ridge (2000).

Figure 4 shows $F_{\mathrm{CO}}$ and $L_{\mathrm{CO}}$ plotted against source bolometric luminosity. Figure 4 a confirms that the force required to drive the outflows, $F_{\mathrm{CO}}$, is approximately 100 times greater than the force available in radiation pressure from stellar photons $\left(L_{\mathrm{bol}} / c\right.$, assuming single scattering) indicated by the solid line, and therefore radiation pressure cannot be the sole driving mechanism for these outflows. A Spearman rank test gives correlation coefficients of 0.55 between flow force and $L_{\mathrm{bol}}$, and 0.61 between flow power and $L_{\text {bol }}$. Neither of these are equivalent to a significant correlation $(2.0 \sigma$ and $2.3 \sigma$ respectively). There are two particularly noticeable outliers which fall well below the dashed line indicating the CB relation in Fig. 4. The ${ }^{13} \mathrm{CO}$ emission from the object NGC 6334B is complex, and may be optically thick even in the wings so the values of $L_{\mathrm{CO}}$ and $F_{\mathrm{CO}}$ will be lower limits. Additionally, this flow extended outside the mapped region due to the poor information available in the literature about the size and exact location of this flow, causing the mass (and therefore dynamics) to be underestimated. These factors could easily increase the measured values of the flow dynamics by up to a factor of 10 , bringing it into line with the scatter of the other objects. There is no similar explanation for the weakness of the flow from IRAS 20188, and this source appears to be driving a genuinely weak outflow. Additionally, there are two sources which appear to be driving particularly powerful outflows, AFGL 437 and AFGL 5157. However, both these flows are within a factor of $\sim 5$ of CB's relation, well within the uncertainties expected in deriving dynamical properties. 

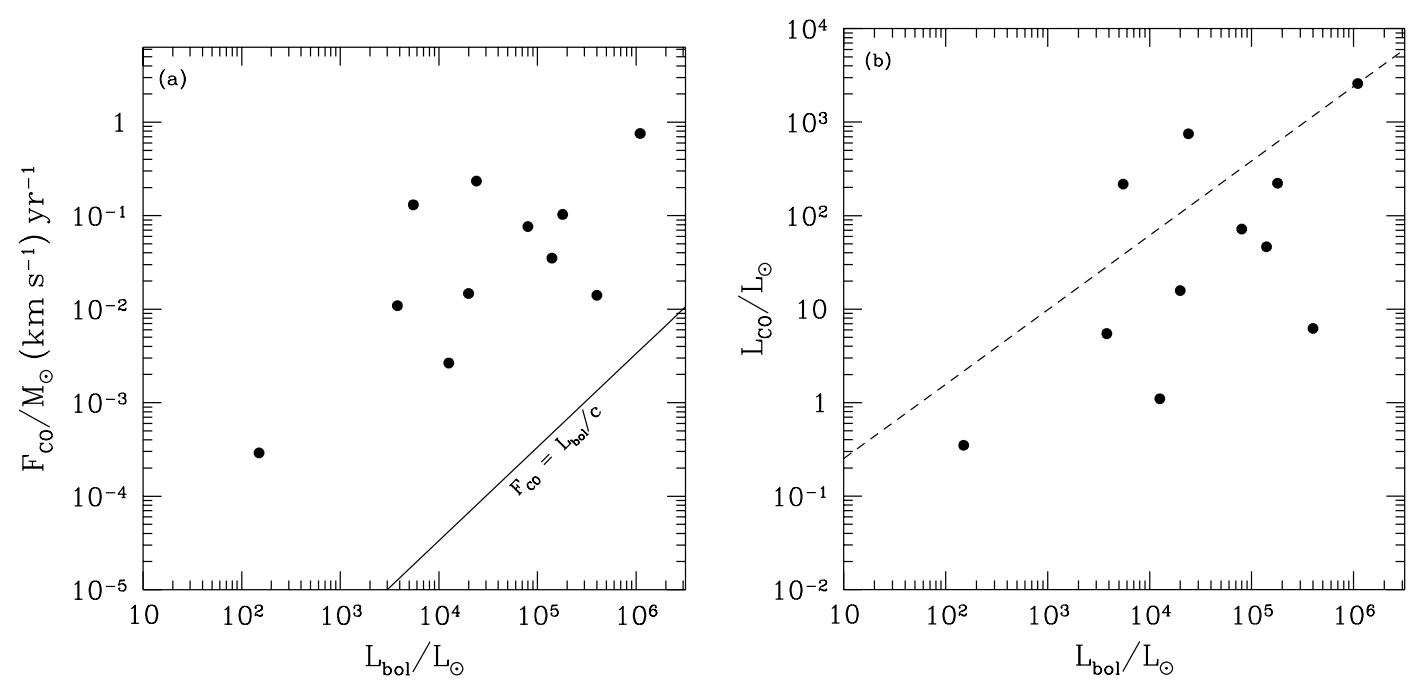

Fig. 4. a) Flow force and b) flow power vs. $L_{\text {bol }}$ for the objects in the single distance sample. The solid line in a) indicates the force available in stellar photons assuming single scattering. The dashed line in $\mathbf{b}$ ) shows the best fit line to the data presented by Cabrit \& Bertout (1992).

Therefore, within the uncertainties involved in calculating dynamical properties from outflows, only the object IRAS 20188 is not consistent with the previously presented relations between outflow force and $L_{\mathrm{bol}}$ and outflow power and $L_{\mathrm{bol}}$.

\subsubsection{Mass outflow rate}

Figure 5 shows the mass outflow rate, $\dot{M}_{\mathrm{f}}=M_{\mathrm{f}} / t_{\text {dyn }}$ plotted against bolometric luminosity. The mass outflow rates calculated for this sample are a factor of $\sim 10$ greater than those derived by Shepherd \& Churchwell (1996b) for objects with a similar bolometric luminosity. This discrepancy is probably due to the different techniques used for determining the the velocity at which the mass is dominated by cloud core emission (low-velocity cut-off in the integration). Masson \& Chernin (1994) and Ridge (2000) have shown that this can significantly influence the measured mass in a flow, and is the largest source of error in mass-determination. Therefore the low-velocity cut-off has to be selected in a consistent manner when comparing samples of objects (see Ridge 2000 for a full discussion of the method adopted here).

There is no significant correlation between mass outflow rate and bolometric luminosity - a Spearman rank test gives a correlation coefficient of 0.49 , equivalent to a $1.7 \sigma$ correlation. This appears to be in contradiction with the results of Shepherd \& Churchwell (1996b). However the relation they present shows a large scatter in $\dot{M}_{\mathrm{f}}$ among the high luminosity objects, and would probably not produce a significant correlation if the objects in their sample with $L_{\mathrm{bol}}<100 L_{\odot}$ were disregarded.

\subsubsection{Mass-velocity relation}

A number of authors have observed a power-law relation between intensity and velocity in the high-velocity wings

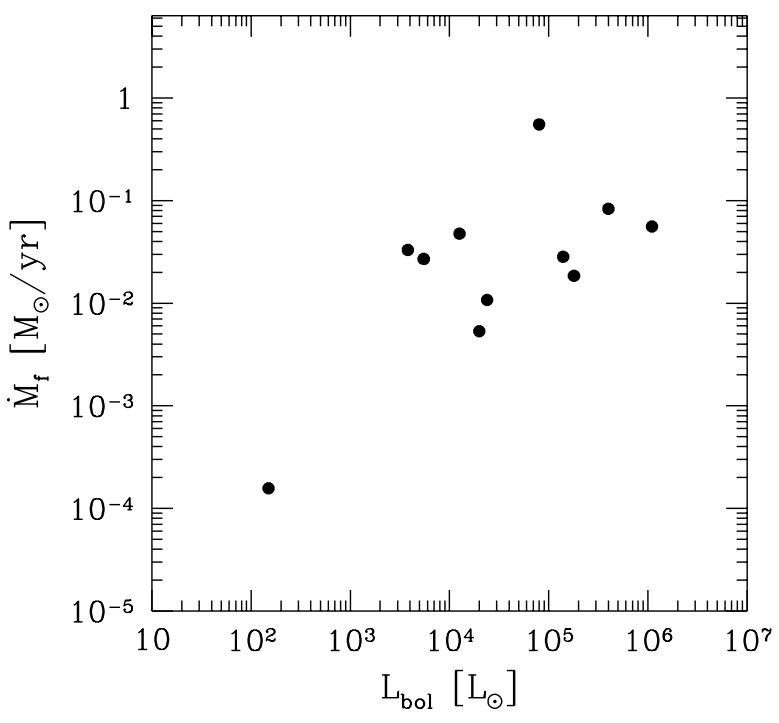

Fig. 5. The mass outflow rate $\dot{M}_{\mathrm{f}}$ versus the bolometric luminosity $L_{\mathrm{bol}}$.

of CO (e.g. Davis et al. 1998; Lada \& Fich 1996). In most of the objects in this sample, at least part of the line profile is well described by a power-law $I \propto V^{-\gamma}$. Least squares fits to the spatially averaged spectra are summarised in Table 5. Where a single power law did not describe the emission over all velocities, two values are given in Table 5, the first describing the behaviour at low velocities $\left(\lesssim 10 \mathrm{~km} \mathrm{~s}^{-1}\right)$ and the second the higher-velocity emission $\left(\gtrsim 10 \mathrm{~km} \mathrm{~s}^{-1}\right)$. The slope for the red wing of the object W75 $\mathrm{N}$ is shown in Fig. 6 as an example. There is large spread in the values of both the low- and highvelocity $\gamma \mathrm{s}$ measured for the flows. The mass-spectrum slope is steeper for the high-velocity emission in all cases except the red wing emission of GGD 27. The blue wing of W3 IRS5 shows particularly unusual behaviour, with the mass-velocity relation steepening to a slope $\sim-12$ at a 


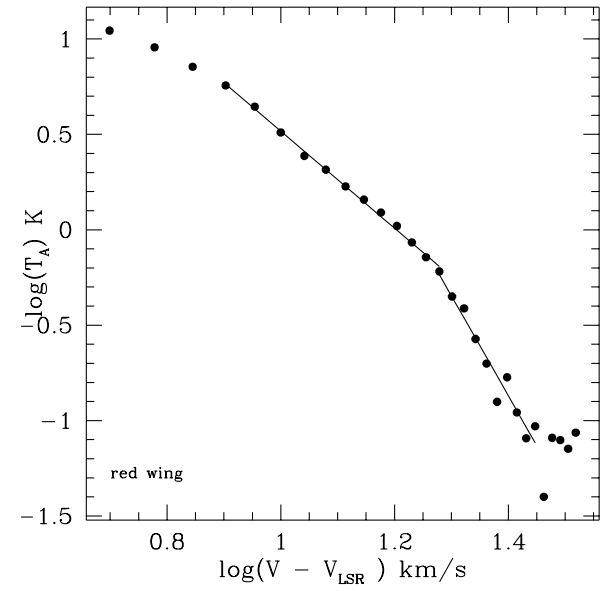

Fig. 6. Spatially averaged intensity vs. velocity for red lobe of $\mathrm{W} 75 \mathrm{~N}$, showing a power law relation between intensity and velocity, breaking at $\sim 18 \mathrm{~km} \mathrm{~s}^{-1}$.

Table 5. The value of $\gamma$, the power-law exponent of the massvelocity relation for the objects in the single-distance sample. Left column gives the value for the blue wing $\left(\gamma_{\text {blue }}\right)$ and right column the value for the red wing $\left(\gamma_{\text {red }}\right)$.

\begin{tabular}{ccc}
\hline Object & $\gamma_{\text {blue }}$ & $\gamma_{\text {red }}$ \\
\hline AFGL 437 & $-5.13 \pm 0.20$ & $-2.86 \pm 0.03$ \\
AFGL 5142 & $-2.40 \pm 0.07$ & $-2.19 \pm 0.04$ \\
AFGL 5157 & $-1.30 \pm 0.01$ & $-1.59 \pm 0.03$ \\
& $-2.24 \pm 0.03$ & $-3.79 \pm 0.14$ \\
S88 B & $-9.06 \pm 0.06$ & $-6.66 \pm 0.09$ \\
GGD 27 & $-2.60 \pm 0.04$ & $-3.57 \pm 0.02$ \\
& & $-1.55 \pm 0.12$ \\
W75 N & - & $-2.54 \pm 0.02$ \\
& - & $-5.23 \pm 0.06$ \\
W3 IRS5 & $-4.99 \pm 0.02$ & $-2.90 \pm 0.04$ \\
& $-12.52 \pm 0.02$ & $-4.15 \pm 0.01$ \\
IRAS 19550 & $-1.91 \pm 0.04$ & $-1.52 \pm 0.03$ \\
IRAS 20188 & $-1.21 \pm 0.01$ & $-3.74 \pm 0.06$ \\
& $-5.07 \pm 0.08$ & \\
NGC 6334I & $-0.98 \pm 0.03$ & $-0.99 \pm 0.06$ \\
& & $-9.23 \pm 0.4$ \\
\hline
\end{tabular}

velocity of $\sim 10 \mathrm{kms}^{-1}$, then showing a small bump between velocities of 14 and $16 \mathrm{~km} \mathrm{~s}^{-1}$ (see Fig. 7).

The low-velocity mass-spectrum slopes observed for the majority of the flows are significantly steeper than the often-quoted value of $\sim 1.7-1.8$ for low-mass YSO outflows (Lada \& Fich 1996). This supports the conclusions of Davis et al. (1998) who also found that outflows from highmass YSOs have a steeper mass-velocity relation than is observed in low-mass flows. They interpreted this as evidence for the more evolved nature of the flows they observed. We do not find a correlation between massspectrum slope and bolometric luminosity however (see Fig. 8a).

The slopes derived here cannot be considered a true mass-velocity relation, as in most cases, the emission is optically thick, and therefore mass is not directly

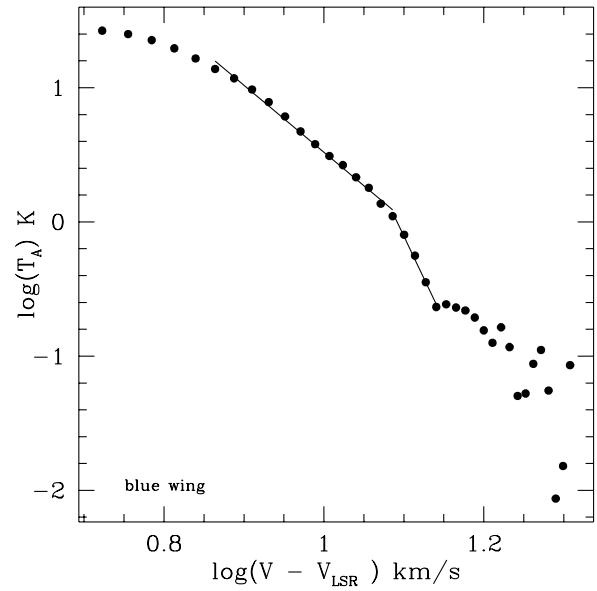

Fig. 7. Spatially averaged intensity vs. velocity for blue lobe of W3 IRS5, showing a power law relation between intensity and velocity, at lower velocities, but a bump at $\sim 16 \mathrm{~km} \mathrm{~s}^{-1}$.

proportional to intensity. The optical depth decreases with velocity (Ridge 2000; Shepherd \& Churchwell 1996b) and therefore the correction is more extreme for the lower velocity emission. This means that the true slope $(\gamma)$ of the mass-velocity relation for these objects would be even steeper. Table 5 also shows that in all but one case (GGD 27), the value of $\gamma$ for the red wing is smaller than $\gamma$ for the blue wing. It is not clear what the cause of this effect is. Optical depth effects would cause red-shifted selfabsorption in infalling material (e.g. Myers et al. 1996).

Figure 8 shows the slopes plotted against bolometric luminosity, dynamical timescale $t_{\text {dyn }}$ (flow age) and wing extent. We do not find the clear separation between $\gamma_{\mathrm{S}}$ measured for the high velocity $\left(\gtrsim 10 \mathrm{~km} \mathrm{~s}^{-1}\right)$ and low velocity emission that was presented by Richer et al. (2000) for a sample of twenty-one outflows spanning a luminosity range from $\sim 0.3-10^{6} L_{\odot}$ compiled from the literature. There is some suggestion that there is an increase in the magnitude of $\gamma$ with luminosity, but there are too few sources in the low-luminosity range to make this conclusive. The spread of $\gamma \mathrm{s}$ does seem to increase with bolometric luminosity, similar to Fig. 4 of Richer et al. (2000). They also proposed that there exists a weak relationship between outflow dynamical age and mass-spectrum slope in high mass YSO outflows, with the slope becoming steeper with age. This is not evident in Fig. 8b, although we only have a relatively narrow range of flow ages so cannot rule this possibility out completely. Figure 8c shows $\gamma$ plotted against $\Delta V$, to test whether the value of $\gamma$ is affected by the detectable width of the line wings. No correlation is present between either the blue- or red-shifted $\gamma \mathrm{s}$ and $\Delta V(\ll 1 \sigma$ in all four cases $)$.

\section{Discussion}

\subsection{The sample}

The objects in this single-distance sample were selected from the catalogue of $\mathrm{Wu}$ et al. (1996). This catalogue 

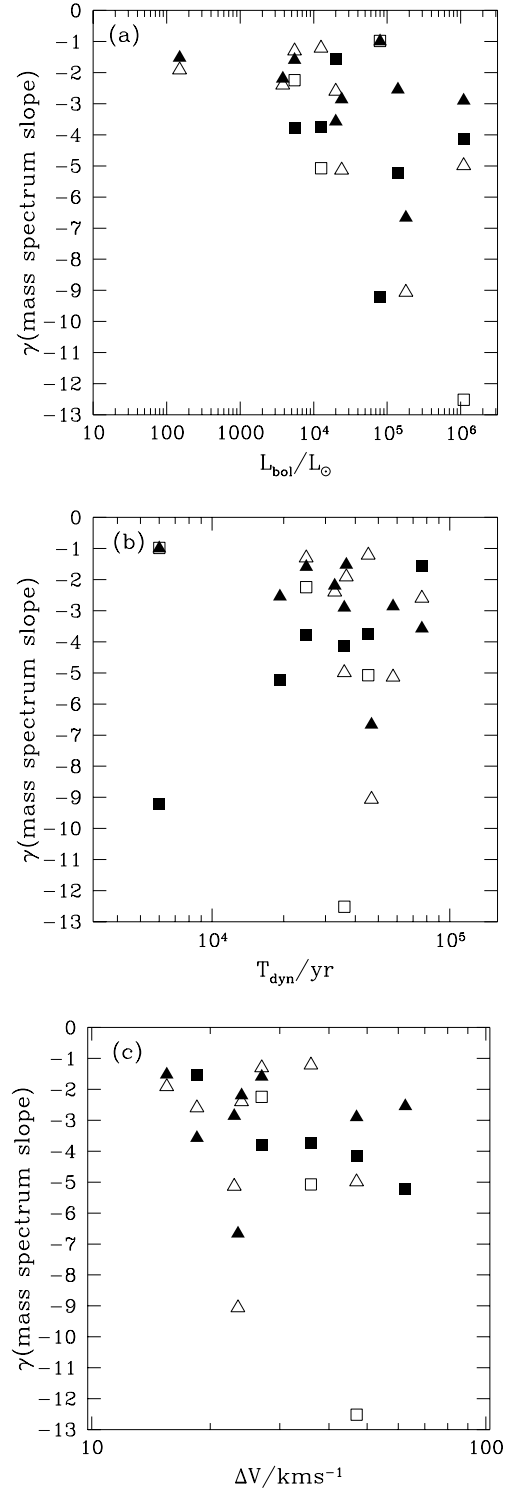

Fig. 8. The slope $\gamma$ of the mass spectrum $M(v)$ plotted as a function of a) source bolometric luminosity $L_{\mathrm{bol}}$, b) dynamical age $t_{\text {dyn }}$ and $\mathbf{c}$ ) wing extent $\Delta V$. Triangles represent $\gamma$ for gas with low velocity $\left(\lesssim 10 \mathrm{~km} \mathrm{~s}^{-1}\right)$ relative to the source, and squares are for gas moving at more than $10 \mathrm{~km} \mathrm{~s}^{-1}$. Filled symbols show $\gamma \mathrm{s}$ measured for red shifted emission, and open symbols show $\gamma \mathrm{s}$ for blue-shifted emission.

was compiled from the literature and includes YSOs identified from their IRAS spectral energy distribution (SED), radio surveys for H II regions (e.g. Wood \& Churchwell) and radio-continuum emission, and other outflow objects discovered either serendipitously or as part of targeted observations towards known YSO candidates. The catalogue cannot therefore be considered complete, and is not volume-limited. Wu et al. do not discuss the possible selection effects in their catalogue. Bearing this in mind, the sample may not be representative of the true YSO population.

In particular, there may be biases in our sample associated with YSOs identified from their IRAS SED and/or existence of radio continuum. This is likely to bias the selection to older YSOs (Class I or later), as many Class 0 objects are still invisible even at far-infrared wavelengths. Outflows found as part of targeted observations of known YSOs may also introduce biases, as it is often the interesting, and therefore probably unusual (strongest), objects that are selected for detailed multi-wavelength study.

However, by selecting objects with a range of luminosities with the same distance, we believe we have achieved a good approximation to a representative sample of the properties of YSO outflows.

\subsection{Correlation between $F_{\mathrm{co}}$ and $L_{\mathrm{bol}}$}

We find a marginal $(2.3 \sigma)$ correlation between outflow power and source luminosity (Sect. 4.2.2). A least squares fit shows a relation of $F_{\mathrm{CO}} \propto L_{\mathrm{bol}}{ }^{0.62 \pm 0.21}$, which is consistent with the slope of $0.69 \pm 0.05$ for the high-luminosity objects in the sample of CB. Our data are offset to lower $L_{\mathrm{CO}}$ compared to Cabrit \& Bertout's data, but again are not inconsistent within the uncertainties. Cabrit \& Bertout selected well-collimated flows for their study, and it is therefore likely to be dominated by outflows from Class 0 sources (Bontemps et al. 1996). However, YSO classes are only defined for low-mass objects and just a handful of high-mass Class 0 candidates have been identified to date (e.g. Molinari et al. 1998). Models of highmass star-formation (e.g. Stahler et al. 2000) predict there may in fact be no such thing as a high-mass protostar, as the stellar birthline intersects the main-sequence for stars of $\gtrsim 10 M_{\odot}$. It is therefore likely that the majority of the sources in our sample are equivalent to Class I objects or later. Bontemps et al. (1996) find a steeper slope $(\sim 1)$ for the Class I sources in their low-intermediate mass $\left(L_{\text {bol }}<100\right)$ sample (consistent with the slope for just the lower-mass $\left(L_{\mathrm{bol}} \lesssim 100\right)$ objects in CB's sample), giving an almost linear relationship between $F_{\mathrm{CO}}$ and $L_{\mathrm{bol}}$, and indicating that all Class I sources have approximately the same outflow efficiency, $F_{\mathrm{CO}} / F_{\mathrm{rad}}$, of $\sim 100$, where $F_{\text {rad }}$ is the radiative momentum flux, equal to $L_{\mathrm{bol}} / c$. Bontemps et al. also find that Class 0 sources have an outflow efficiency of $\sim 1000$, i.e. a factor of $\sim 10$ more efficient than Class Is.

The outflows in our sample show a range of outflow efficiencies (Fig. 9) from $\sim 10$ to $\sim$ few times $10^{3}$ (i.e. 10 to 1000 times the force due to radiation pressure assuming single scattering), too large for radiation pressure to be important in driving these flows, even if multiple scattering is present. If outflow efficiency is an indicator of YSO class (and therefore age) as Bontemps et al. suggest based on their observational results, then the single-distance sample must contain a mixture of Class 0 and Class I sources. There is a marginal $(2.1 \sigma)$ anti-correlation between outflow efficiency and bolometric luminosity in our sample. This may be an evolutionary effect, as massive YSOs are thought to evolve more rapidly, and it is therefore likely 


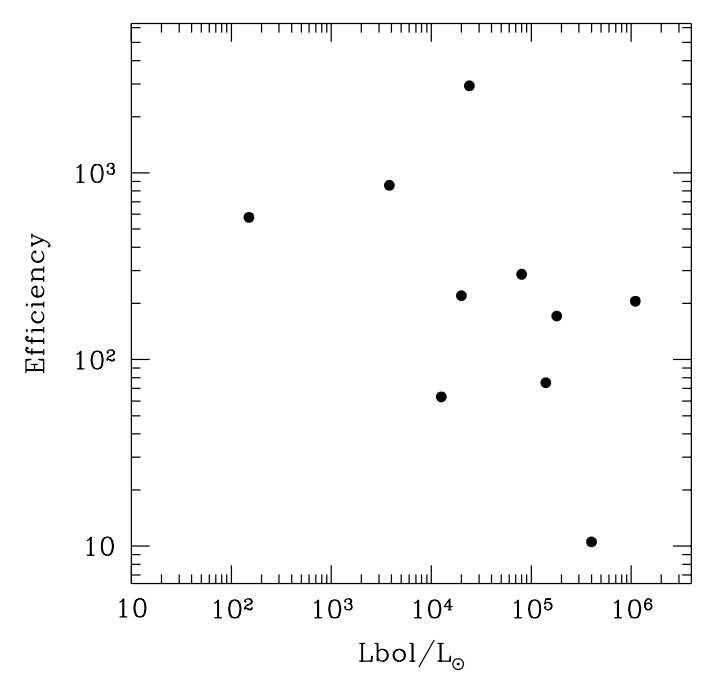

Fig. 9. Outflow efficiency versus $L_{\text {bol }}$ for the objects in the single distance sample.

that on average we are seeing them at a later stage in their pre-main-sequence evolution.

The outflow efficiencies agree well with other previous works covering several decades in luminosity (e.g. Rodríguez et al. 1982; Bally \& Lada 1983; Lada 1985; Levreault 1988) and with CB who found an efficiency of $\sim 250$ for their entire sample, while for the lower luminosity sources alone the efficiency was $\sim 1000$.

\subsection{Collimation}

The flows in the single distance sample are much less well collimated than the molecular outflows from their low-mass counterparts (collimation factors, $R_{\text {coll }}=$ $R_{\max } / R_{\min }$, of $\sim 1-2$ in the high-mass sample as opposed to $\gtrsim 6$ in low-mass objects, Lada 1985). There could be several reasons which could explain the relatively poor collimation of these flows. One possibility is that in some cases of high-mass outflows we are not seeing the outflow from a single star, but the superposition of several highly-collimated outflows from a cluster of young stars. High-mass star forming regions are also generally further away than the well-studied low-mass star forming regions, and therefore the hypothesis of multiple outflows requires high-resolution observations in both the mid-infrared (to resolve the stellar cluster) and in ${ }^{12} \mathrm{CO}$ (to resolve the outflows) in order to be tested thoroughly. The ${ }^{12} \mathrm{CO}$ observations would require mm-interferometry well beyond the capabilities of current facilities. Alternatively, the molecular outflow may be tracing the density structure close to the YSO. For instance, Mellema \& Frank (1997) have shown that aspherical bubbles can form from the interaction of a central YSO wind with a toroidal circumprotostellar density distribution. Observations of cloud core morphologies (Ridge 2000) do not seem to indicate a toroidal morphology however.

\subsection{Mass-velocity relation}

We find an extremely steep mass-velocity relation in a number of the flows in the sample, indicating a high massfraction at lower velocities. This has previously been interpreted as evidence for the more evolved nature of highmass outflows, as the majority of material has had time to slow down after its initial acceleration due to either prompt or turbulent entrainment (e.g. Davis et al. 1998). However it is also possible that high-mass outflows are accelerated by a different mechanism, with the majority of the momentum transferred to large amounts of slowmoving material in the flow.

Six of the eleven objects in the sample show distinct breaks in the power-law between low- and high- velocity gas, as has been observed in some low-mass flows (e.g. Lada \& Fich 1996). Richer et al. (2000) found that the slopes $(\gamma \mathrm{s})$ for the low-velocity gas are similar in sources of all luminosities $(0<\gamma<2.5)$, and interpreted this as further evidence that a common acceleration mechanism operates over nearly six decades in $L_{\text {bol }}$. Richer et al. also found a clear separation between $\gamma \mathrm{s}$ in high- and lowvelocity gas, interpreting this as evidence for two distinct outflow velocity components, corresponding to a recently accelerated component and a slower, coasting component. We found a large range of $\gamma$ s for both the low- and highvelocity gas, and therefore my data does not support these conclusions.

Models of jet-driven molecular outflows (e.g. Downes \& Ray 1999; Smith et al. 1997) have tried to reproduce the power-law of $\sim 1.7$ observed by e.g. Lada \& Fich (1996) in low-mass flows with some success, and the hydrodynamic simulation by Smith et al. (1997) also predicted the change in slope at high velocities, due to a jet-bow shear layer consisting of molecules which survived the jet terminal shock. Smith et al. also predict that $\gamma$ should steepen over time, possibly due to the collection of a reservoir of low-velocity gas. Unlike Richer et al. we do not find any correlation between outflow age (dynamical timescale) and the magnitude of $\gamma$, but the data contain only a narrow range of flow ages, so we cannot rule this possibility out completely.

Alternatively, Downes \& Ray (1999) state that in their model, the molecular fraction in the jet has a significant influence on the value of $\gamma$ their model predicts, with $\gamma$ increasing with decreasing molecular abundance in the jet. If this is the case, then the high values of $\gamma$ found for these high-mass outflows would imply a small molecular fraction in the entraining jet, and the large range of $\gamma \mathrm{s}$ $(\sim 1$ to $\sim 12)$ would suggest that a large range of molecular abundances is possible in protostellar jets. However, their simulation of an atomic jet, which should give an upper limit to the value of $\gamma$ their model can produce, gives $\gamma$ of 3.75 , much less than the observed values even for the low-velocity material, and it is therefore unclear whether the molecular abundance of the jets has any role to play in the slope of the mass-velocity relation. 


\subsection{The Hubble law}

The existence of a Hubble-like relation $(v \propto r)$ for molecular outflows (e.g. Lada \& Fich 1996) has been challenged by Padman et al. (1997), who state that such a law may apply locally at particular bow shocks, and this could be interpreted as a more global phenomenon in small or incompletely-mapped sources. This conclusion is supported by the numerical simulations presented by e.g. Downes \& Ray (1999) which show that the Hubble law is almost certainly a local effect in the vicinity of a bow shock. If a Hubble law was more global, it would be difficult to reconcile with jet-driven models which accelerate the molecular material in situ rather than it originating at the source. The observations presented here show no evidence for a Hubble law in any of the high-mass objects studied (see also the channel maps presented in Ridge 2000).

Models such as the simulations of Smith et al. (1997) reproduce Hubble-like position-velocity diagrams well. However, the lack of observational evidence for a global Hubble-like flow puts this phenomenon in doubt.

\section{Conclusions}

Using a sample of known outflow objects at a single distance, we have investigated the physical and dynamical properties of intermediate and high-mass YSOs. Our main results are:

- We find a similar relation between $F_{\mathrm{CO}}$ and $L_{\mathrm{bol}}$ as previous studies (CB , Shepherd \& Churchwell 1996b) but with a large scatter;

- We find a much steeper mass-velocity relation than has been observed in low-mass outflows. There is also a large range of values of $\gamma$, from $\sim-1$ to $\sim-12$;

- None of the objects studied show a "Hubble-law" like relation.

We have found that several of the often-quoted observational results for low-mass YSO outflows are not applicable to high-mass stars. To attempt to understand fully high mass star formation and their outflows, more extensive statistical studies need to be undertaken, as we have shown that the extrapolation of outflow properties from low-mass stars do not necessarily agree with observations of high mass stars.

Acknowledgements. We would like to thank John Porter for his comments on earlier versions of this manuscript, Chris Collins for useful discussions and the referee Rafael Bachiller for useful suggestions. NAR acknowledges the support of a PPARC studentship. The James Clerk Maxwell Telescope is operated by the Joint Astronomy Centre on behalf of the Particle Physics and Astronomy Research Council of the UK, The Netherlands Organisation for Scientific Research and the National Research Council of Canada. The National Radio Astronomy Observatory is a facility of the National Science Foundation, operated under cooperative agreement by Associated Universities, Inc.

\section{References}

Arquilla, R., \& Goldsmith, P. F. 1984, ApJ, 279, 664

Aspin, C., Puxley, P. J., Blanco, P. R., et al. 1994, A\&A, 292, L9

Bachiller, R. 1996, ARA\&A, 34, 111

Bachiller, R., \& Cernicharo, J. 1990, A\&A, 239, 276

Bally, J., \& Lada, C. J. 1983, ApJ, 265, 824

Bontemps, S., André, P., Terebey, S., \& Cabrit, S. 1996, A\&A, 311,858

Cabrit, S., \& Bertout, C. 1992, A\&A, 261, 274

Carpenter, J. M., Snell, R. L., \& Schloerb, F. P. 1990, ApJ, 362,147

Davis, C. J., Smith, M. D., \& Moriarty-Schieven, G. H. 1998, MNRAS, 299, 825

Dickel, H. R., Dickel, J. R., \& Wilson, W. J. 1977, ApJ, 217, 56

Downes, T. P., \& Ray, T. P. 1999, A\&A, 345, 977

Dyck, H. M., \& Lonsdale, C. J. 1979, AJ, 84, 1339

Gómez, J. F., Torrelles, J. M., Estalella, R., et al. 1992, ApJ, 397,492

Gyulbudaghian, A. L., Glushkov, I. I., \& Denisiuk, A. E. 1978, ApJ, 224, L137

Henning, T., Schreyer, K., Launhardt, R., \& Burkert, A. 2000, A\&A, 353, 211

Higgs, L. A. 1971, MNRAS, 153, 315

Hodapp, K. W. 1994, ApJS, 94, 615

Hunter, T. R., Taylor, G. B., Felli, M., \& Tofani, G. 1994, A\&A, 284, 215

Hunter, T. R., Testi, L., Taylor, G. B., et al. 1995, A\&A, 302, 249

Imai, H., Kameya, O., Sasao, T., et al. 2000, ApJ, 538, 751

Kleinmann, S. G., Sargent, D. G., Gillett, F. C., Grasdalen, G. L., \& Joyce, R. R. 1977, ApJ, 215, L79

Koo, B., Lee, Y., Fuller, G. A., et al. 1994, ApJ, 429, 233

Kraemer, K. E., \& Jackson, J. M. 1995, ApJ, 439, L9

Kutner, M. L., \& Ulich, B. L. 1981, ApJ, 250, 341

Lada, C. J. 1985, ARA\&A, 23, 267

Lada, C. J., \& Fich, M. 1996, ApJ, 459, 638

Langer, W. D., \& Penzias, A. A. 1990, ApJ, 357, 477

Levreault, R. M. 1988, ApJ, 330, 897

Little, L. T., Bergman, P., Cunningham, C. T., et al. 1988, A\&A, 205, 129

Loughran, L., McBreen, B., Fazio, G. G., et al. 1986, ApJ, 303, 629

Masson, C. R., \& Chernin, L. M. 1994, in ASP Conf. Ser. 65, Clouds, Cores, and Low Mass Stars, 350

McCutcheon, W. H., Sandell, G., Matthews, H. E., et al. 2000, MNRAS, 316, 152

Mellema, G., \& Frank, A. 1997, MNRAS, 292, 795

Molinari, S., Testi, L., Brand, J., Cesaroni, R., \& Palla, F. 1998, ApJ, 505, L39

Moore, T. J. T. 1989, Ph.D. Thesis, Univ. of Edinburgh

Moore, T. J. T., Mountain, C. M., \& Yamashita, T. 1991a, MNRAS, 248, 79

Moore, T. J. T., Mountain, C. M., Yamashita, T., \& McLean, I. S. 1991b, MNRAS, 248, 377

Myers, P. C., Mardones, D., Tafalla, M., Williams, J. P., \& Wilner, D. J. 1996, ApJ, 465, L133

Padman, R., Bence, S., \& Richer, J. 1997, in IAU Symp. 182, Herbig-Haro Flows and the Birth of Low Mass Stars, vol. 182,123 
Phillips, J. P., \& Mampaso, A. 1991, A\&AS, 88, 189

Richards, P. J., Little, L. T., Heaton, B. D., \& Toriseva, M. 1987, MNRAS, 228, 43

Richer, J. S., Shepherd, D. S., Cabrit, S., Bachiller, R., \& Churchwell, E. 2000, in Protostars and Planets IV, ed. V. Mannings, A. P. Boss, \& S. S. Russell (Univ. of Arizona Press), vol. 1, 867

Ridge, N. A. 2000, Ph.D. Thesis, Liverpool John Moores University

Roberts, W. W. 1972, ApJ, 173, 259

Rodríguez, L. F., Carral, P., Moran, J. M., \& Ho, P. T. P. 1982, ApJ, 260, 635

Sandell, G. 2000, A\&A, 358, 242

Schneps, M. H., Ho, P. T. P., Barrett, A. H., \& Martin, R. N. 1978, ApJ, 221, 124

Shepherd, D. S., \& Churchwell, E. 1996a, ApJ, 457, 267

Shepherd, D. S., \& Churchwell, E. 1996b, ApJ, 472, 225

Smith, M. D., Suttner, G., \& Yorke, H. W. 1997, A\&A, 323, 223
Snell, R. L., Huang, Y., Dickman, R. L., \& Claussen, M. J. 1988, ApJ, 325, 853

Stahler, S. W., Palla, F., \& Ho, P. T. P. 2000, in Protostars and Planets IV, ed. V. Mannings, A. P. Boss, \& S. S. Russell (University of Arizona Press), 327

Torrelles, J. M., Eiroa, C., Miranda, L. F., et al. 1992b, ApJ, 384,528

Torrelles, J. M., Gómez, J. F., Anglada, G., et al. 1992a, ApJ, 392,616

Verdes-Montenegro, L., Torrelles, J. M., Rodríguez, L. F., et al. 1989, ApJ, 346, 193

Weintraub, D. A., \& Kastner, J. H. 1996, ApJ, 458, 670

Willner, S. P. 1977, ApJ, 214, 706

Wood, D. O. S., \& Churchwell, E. 1989, ApJS, 69, 831

Wu, Y., Huang, M., \& He, J. 1996, A\&AS, 115, 283

Wynn-Williams, C. G., Becklin, E. E., \& Neugebauer, G. 1972, MNRAS, 160, 1

Yamashita, T., Sato, S., Nagata, T., et al. 1987, A\&A, 177, 258 